\title{
Mémoires quantiques
}

\author{
Thierry CHANELIÈRE \\ Chargé de recherche au CNRS, Laboratoire Aimé Cotton, Orsay \\ Julien LAURAT \\ Professeur à l'université Pierre et Marie Curie, Laboratoire Kastler Brossel, Paris \\ thierry.chaneliere@u-psud.fr
}

L'apport de l'optique à l'informatique et aux télécommunications modernes en général est indéniable. Les fibres optiques couvrent la planète et arrivent maintenant jusqu'à nos maisons. Nos ordinateurs personnels ne sont pas en reste puisque le stockage optique de l'information dans les disques Blu-ray par exemple, descendants directs des CD-ROM, sont une référence en densité de stockage. La transposition d'un tel schéma de stockage pour des photons porteurs d'information quantique n'est malheureusement pas possible. La piste d'un CD est une suite de bits 0 et 1 où l'on a perdu la superposition cohérente $\alpha|0\rangle+\beta|1\rangle$ de deux états comme l'autorise la mécanique quantique. On parle alors de bit quantique, ou plus brièvement qubit (voir aussi les autres articles de ce dossier).

L e besoin d'une mémoire qui préserve cette superposition est crucial en information quantique. En effet, la communication quantique sur de longues distances requiert des mémoires qui assurent la synchronisation des échanges. La sécurité d'une transmission cryptée quantiquement est alors garantie tout au long de la chaîne de transmission, y compris pendant le stockage [1]. Maîtriser l'interaction de façon réversible entre un photon et un système matériel est aussi un défi fondamental particulièrement stimulant puisqu'il s'agit de préserver aussi bien à l'écriture qu'à la lecture une superposition quantique dont la force et la faiblesse sont précisément sa fragilité.

Lorsque l'information est portée par un quantum de lumière, le photon, il est naturel de s'intéresser comme support matériel de stockage au quantum de matière que représente un atome. Un atome présente en général une série d'états quantiques discrets dont on peut isoler une paire notée par exemple $|g\rangle,|s\rangle$. Le processus de mémorisation consiste à mettre l'atome dans l'état $\alpha|g\rangle+\beta|s\rangle$ en enregistrant l'état du qubit incident puis à le relire pour régénérer à un instant ultérieur l'état $\alpha|0\rangle+\beta|1\rangle$ du photon.

Après avoir présenté sommairement différents systèmes atomiques considérés comme de bons supports matériels pour l'information quantique, nous discuterons des protocoles qui permettent cette séquence d'opérations écriture/lecture. Comment stocker par exemple un qubit photonique dans un atome unique? Nous détaillerons ensuite une solution alternative consistant à utiliser un large ensemble d'atomes. Cette dernière approche a été très féconde de réalisations expérimentales ces dernières années et ouvre la voie à un stockage « en parallèle » de plusieurs qubits.

\section{Différents systèmes atomiques comme support de stockage}

Les systèmes atomiques sont largement utilisés comme support de mémoire quantique [2]. Il est important ici de définir les termes. On entend par atome un système simple du point de vue de la mécanique quantique. L'atome est alors décrit par un nombre restreint d'états discrets dont on pourra extraire deux états $|\mathrm{g}\rangle$ et $|\mathrm{s}\rangle$ particuliers et bien isolés. Cette simplicité structurale assure aussi une bonne préservation de l'information lorsqu'elle est portée par deux états internes. D'un point de vue classique, les atomes sont de bons oscillateurs que l'on utilise internationalement en métrologie comme référence de temps. Cette capacité à conserver une oscillation sans perte d'amplitude ou de phase se traduira par un long temps de mémorisation. Lorsqu'on s'intéresse au système à deux niveaux génériques $|g\rangle,|s\rangle$, on parle alors de temps de cohérence. C'est un paramètre clé dans le choix du système matériel. Deux grandes familles sont utilisées pour faire des mémoires quantiques.

Tout d'abord, les atomes alcalins sont des systèmes de référence pour l'interaction lumière-matière. Sous forme de vapeur, l'ensemble est suffisamment dilué pour que les atomes n'interagissent pas entre eux. Les collisions sont en effet délétères pour la préservation de l'information, elles se traduisent par exemple par une réduction du temps de cohérence de l'état de superposition quantique. La vitesse des particules est cependant élevée pour des atomes à température ambiante. L'information est alors rapidement perdue, car l'atome sort du champ de vision que représente le faisceau laser qui porte le qubit photonique. Les outils modernes de la physique atomique apportent une solution avec le refroidissement et le piégeage par laser qui permettent de figer et de maintenir aussi bien une vapeur qu'un atome unique empêchant la mémoire de 
s'envoler littéralement. Les atomes froids ont ainsi permis des réalisations remarquables que nous présenterons ensuite.

Des solutions alternatives aux alcalins sont les solides dopés. Les atomes luminescents sont alors solidement arrimés dans une matrice cristalline transparente (figure 1). Cet arrimage est en général problématique pour le dopant qui se trouve couplé à l'environnement par l'intermédiaire de ses voisins particulièrement proches à cause de la densité du solide. Le temps de cohérence est généralement fortement réduit... sauf à quelques exceptions près. Les ions de terre rare supportent très bien cette inclusion et conservent leurs propriétés de cohérence en environnement cristallin. Les états internes des lanthanides impliquent des électrons $4 f$ qui ne participent pas à l'assemblage cristallin leur conférant des propriétés proches des gaz gelés.

Après cette courte revue, nous allons voir comment il est possible d'écrire, de conserver et de relire l'information entre qubit photonique et atomique.

\section{Un atome pour mémoire}

La méthode à première vue la plus directe pour stocker un photon unique est d'utiliser un atome unique. L'atome absorberait le photon puis le réémettrait en se désexcitant. Cependant, cette technique est peu efficace et peu contrôlable : l'interaction est très peu probable, et l'atome se désexcite de manière aléatoire et dans toutes les directions rendant finalement difficile la collecte du photon réémis. Cependant, une solution existe : elle consiste à placer l'atome dans une cavité optique de très grande finesse pour atteindre un régime dit de couplage fort. C'est ce que l'on appelle l'électrodynamique quantique en cavité, un domaine de l'optique quantique qui a permis de développer une connaissance très fine de l'interaction lumière-matière. Les travaux de Serge Haroche dans le domaine micro-onde, qui ont conduit au prix Nobel de 2012, sans oublier les expériences fondatrices de Jeff Kimble au California Institute of Technology (Caltech) dans le domaine optique, s'inscrivent dans cet effort. Les développements obtenus ces dernières années constituent de véritables tours de force : un atome unique est refroidi par laser et suspendu au centre d'une cavité qui doit être très courte (quelques centaines de micromètres) et dont les miroirs doivent être fortement réfléchissants afin que la lumière circulant entre eux puisse interagir de nombreuses fois

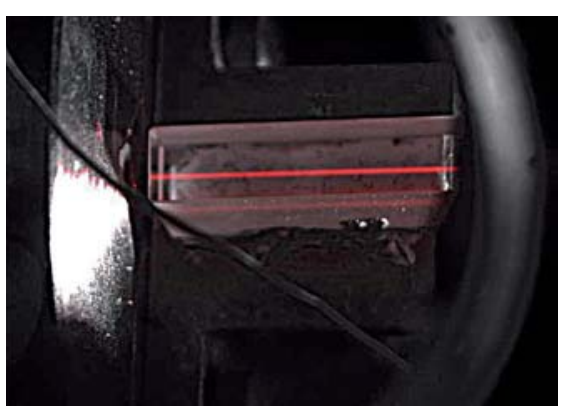

Figure 1. Un cristal pour mémoire : fluorescence de I'ion thulium lorsqu'il est inséré dans une matrice transparente de grenat d'yttrium et d'aluminium, $\mathrm{Y}_{3} \mathrm{Al}_{5} \mathrm{O}_{12}$ (YAG). Le cristal mesure $8 \mathrm{~mm}$ de long et est vu à travers le hublot d'un cryostat qui le maintient à basse température $(2 \mathrm{~K})$. avec l'atome (typiquement quelques dizaines à quelques centaines de milliers d'allers-retours). Dans ces conditions, le photon à stocker peut être absorbé avec une grande probabilité et réémis plus tard dans la direction parfaitement définie par la cavité optique.

Comment stocker cependant un qubit, c'est-à-dire une superposition d'états, dans un seul atome? Intéressons-nous d'un peu plus près à la physique du processus de stockage (figure 2). Très souvent, les deux niveaux énergétiques $|\mathrm{g}\rangle$ et $|\mathrm{s}\rangle$ dans lesquels on souhaite stocker l'information sont deux sous-niveaux qui permettent d'avoir des temps de cohérence longs mais qui sont séparés par une énergie très inférieure à celle du photon à stocker. Un troisième niveau, un niveau excité $|e\rangle$, est donc nécessaire pour qu'il y ait interaction : le photon à stocker est alors résonant avec la transition $|\mathrm{g}\rangle \rightarrow|\mathrm{e}\rangle$ et peut être absorbé si l'atome est initialement préparé dans l'état $|g\rangle$. Afin de permettre un transfert cohérent de l'information, un laser 
auxiliaire, dit de contrôle et qui adresse la seconde transition optique, $|s\rangle \rightarrow|e\rangle$, est nécessaire. II se forme ainsi un système dit en $\Lambda$ où les deux niveaux de stockage sont connectés via le niveau excité. Une fois le transfert effectué, le faisceau de contrôle est éteint et l'atome est figé dans l'état que I'on souhaitait. La lecture se fait ensuite à la demande en le rallumant.

Une première expérience dans cette direction a eu lieu en 2007 au Caltech puis, en 2012, l'équipe de Gerd Rempe au Max Planck Institute de Garching a pu démontrer un réseau élémentaire reposant sur cette approche. Un premier atome piégé émettait un qubit qui était ensuite stocké dans un second système similaire 60 mètres plus loin, avant d'être relu quelques microsecondes plus tard. Aujourd'hui, les recherches dans ce domaine se poursuivent et se concentrent de plus en plus sur les structures nanophotoniques qui permettent un confinement encore plus important de la lumière. Le couplage se fait alors entre une onde évanescente et un atome unique piégé à proximité de la surface. Microtores, nanofibres, ou encore cristaux photoniques sont quelques exemples de systèmes très étudiés actuellement. La possibilité dans le futur d'intégrer un grand nombre de ces systèmes sur puces est l'une des motivations.

\section{Un ensemble pour mémoire}

Une alternative existe à I'utilisation d'atomes uniques. De manière surprenante, les chercheurs ont ainsi développé ces dernières années des protocoles permettant d'utiliser des ensembles d'atomes identiques pour stocker des photons uniques. Le grand nombre d'atomes, plusieurs millions, permet d'augmenter la probabilité d'absorber le photon sans nécessiter de cavité. De plus, lors de la lecture, la lumière est réémise dans une direction bien déterminée. C'est la force du collectif ! Les atomes mis en jeu se comportent en effet comme autant d'émetteurs synchronisés, tout comme le fait un réseau d'antennes. Les ensembles d'atomes peuvent prendre des formes variées : vapeurs chaudes emprisonnées dans une cellule de verre, atomes froids piégés par laser, ions dans une matrice cristalline...

\section{La lumière arrêtée}

Une première méthode de stockage dans de tels ensembles repose sur la technique dite de « transparence induite électromagnétiquement ». Cette technique permet de ralentir la lumière puis de la stopper, avant de la relire à la demande (figure 3). À nouveau, un système énergétique à trois niveaux est utilisé et l'opération est ainsi assistée par un faisceau de contrôle. En l'absence de ce laser additionnel, la lumière à stocker est tout simplement absorbée par les millions d'atomes mis en jeu. Le milieu est totalement opaque. Cependant, en allumant ce faisceau auxiliaire, ses propriétés changent drastiquement : l'ensemble d'atomes devient transparent, d'où le nom de la technique. La vitesse de la lumière est aussi très fortement réduite. Ce phénomène surprenant de « lumière lente » a été observé pour la première fois au cours des années 90 . Le groupe de Lee Hau à Harvard montra ainsi une vitesse de la lumière comparable à celle d'une bicyclette ! Cette réduction de vitesse entraîne une compression spatiale de l'impulsion lumineuse dans l'ensemble atomique. En éteignant alors le faisceau de contrôle, la lumière peut être totalement stoppée : l'information qu'elle portait est intégralement transférée aux atomes, prête à être relue si le faisceau est rallumé. En 2005, plusieurs groupes américains réussirent ainsi à stocker un photon unique en suivant cette méthode, expériences rapidement suivies par le stockage de qubits en polarisation.

Cette année, l'équipe d'optique quantique du Laboratoire Kastler Brossel (LKB) à Paris a également utilisé cette méthode pour démontrer le stockage de bits quantiques encodés cette fois-ci dans le moment angulaire orbital de la lumière, c'est-à-dire dans I'hélicité du front d'onde (figure 4) [3]. Ce degré de liberté offre la possibilité de transmettre de l'information à haut débit en multiplexant celle-ci dans de nombreux modes spatiaux qui sont autant de canaux de communication.

\section{ANALYSEUR DE SPECTRE OPTIQUE OSA20 NOUVEaU}

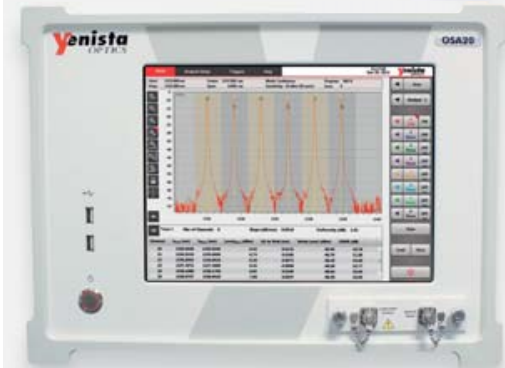

Caractéristiques clés

- Plage : 1250 - 1700 nm

- Résolution : 20 pm

- Vitesse max : $2000 \mathrm{~nm} / \mathrm{s}$

- Courbe de 225.000 points

- Référence $\lambda$ : Acétylène

- Ecran tactile multipoint

- Interfaces externes :

- USB2 et USB3

- Ethernet

- Trigger IN \& OUT

- GPIB

- Modes d'utilisation par application et librairie de fonctions d'analyse

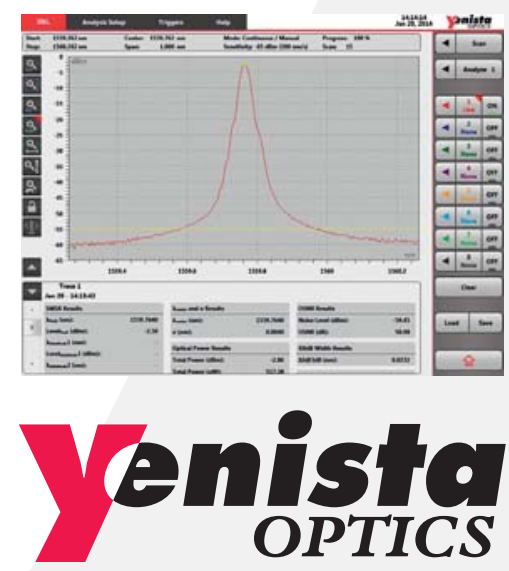

$$
\begin{gathered}
\text { Tél. : +33 (0)29648 } 3716 \\
\text { sales-emea@yenista.com } \\
\text { www.yenista.com }
\end{gathered}
$$


Impulsion à stocker Ensemble d'atomes

ÉCRITURE

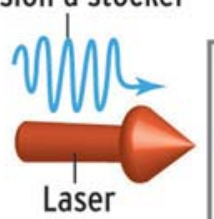
de contrôle
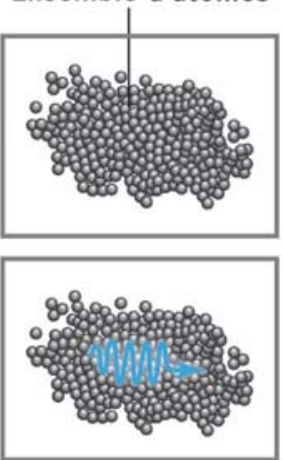

STOCKAGE

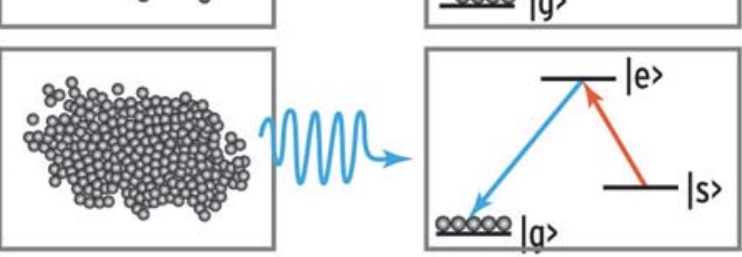

Figure 3. Ralentir et arrêter la lumière dans un ensemble atomique. L'impulsion à stocker est envoyée sur un large ensemble d'atomes en même temps qu'un laser de contrôle. La vitesse de la lumière est fortement réduite et l'impulsion est comprimée spatialement dans l'ensemble. Le faisceau de contrôle est alors éteint et l'information transférée aux atomes. La mémoire est ensuite lue à la demande en allumant de nouveau le laser de contrôle. Là encore, cette méthode utilise un système énergétique en $\Lambda$ et l'information est stockée dans une cohérence atomique entre les deux sous-niveaux $|\mathrm{g}\rangle$ et $|\mathrm{s}\rangle$.

Alors qu'un photon ne peut transporter qu'un seul bit quantique avec sa polarisation, qui ne peut prendre en effet que deux valeurs possibles (horizontale ou verticale), le moment orbital angulaire permet de définir une base de dimension infinie, les modes de Laguerre-Gauss par exemple, et ainsi de transporter un grand nombre de bits quantiques sur un seul photon. L'équipe parisienne a montré

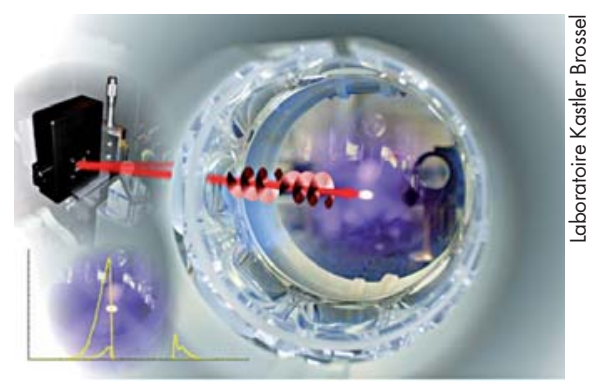

Figure 4. Stocker le twist d'un photon dans une mémoire quantique à atomes froids. Par réflexion sur un modulateur spatial à cristaux liquides, une superposition de deux hélicités différentes du front $d^{\prime}$ 'onde est inscrite dans une impulsion de lumière au niveau du photon unique. L'impulsion est ensuite ralentie puis stockée dans un ensemble de plusieurs millions d'atomes froids de césium par la méthode dite de transparence induite électromagnétiquement. Quelques microsecondes plus tard, à la demande, la lumière est réémise en conservant l'information quantique initialement encodée. qu'il était possible de stocker de tels états dans une mémoire reposant sur un large ensemble d'atomes froids. Un seul ensemble atomique a été nécessaire dans cette expérience montrant ainsi la possibilité de réaliser une mémoire spatialement multimode. Le stockage d'une centaine de bits quantiques dans ce système semble désormais réaliste.

Différents paramètres permettent de caractériser une mémoire quantique : le temps de vie de la mémoire, l'efficacité de stockage et de lecture, ou encore la possibilité de multiplexage temporel ou spatial comme démontrée dans l'expérience du LKB. Des temps de stockage atteignant plusieurs centaines de millisecondes ont été récemment démontrés, nécessitant des atomes très froids piégés optiquement et utilisant des configurations insensibles aux champs magnétiques ou aux déplacements lumineux inhomogènes induits par les faisceaux de piégeage. L'efficacité quant à elle dépend du nombre d'atomes mis en jeu, mais aussi de la préparation efficace du système atomique. Des valeurs proches de $80 \%$ ont été récemment atteintes. Cependant, aucune expérience n'a encore réussi à rassembler toutes ces performances dans un seul système.

\section{L'écho d'un photon}

Une autre méthode utilisant de larges ensembles atomiques utilise la technique " d'écho de photon ». L'écho de photon a largement été utilisé dans les milieux à fort élargissement inhomogène comme les cristaux dopés présentés précédemment pour mesurer le temps de cohérence. La technique a été adaptée plus récemment comme protocole de mémoire quantique.

Pour expliquer l'écho de photon, on peut s'appuyer sur l'image classique d'un atome comme oscillateur. Une première impulsion courte excite uniformément un ensemble inhomogène. Chaque oscillateur pris individuellement vibre comme un ressort stimulé de façon impulsionnelle. À cause de l'élargissement inhomogène, les fréquences propres de vibration étant différentes, les oscillations se déphasent rapidement. Pour compenser ce déphasage primordial, on utilise une seconde impulsion forte dite de rephasage. Cette impulsion dont le profil temporel est optimisé inverse instantanément la phase d'oscillation. Le déphasage se mue alors en rephasage. La suite apparaît comme le renversement temporel du processus d'absorption. L'évolution se termine par une remise en phase des oscillations et l'émission d'un écho (figure 5). Ce dernier traduit simplement le caractère constructif de l'oscillation au moment du rephasage. Encore la force du collectif ! L'opération d'absorption, de déphasage et rephasage doit se dérouler pendant le temps de cohérence où chaque atome individuel oscille à une fréquence certes différente de son voisin, mais parfaitement définie (sans saut de phase intempestif par exemple). Cette séquence est celle d'une mémoire optique où la première impulsion et l'écho correspondent au signal incident et à sa relecture respectivement. Même si les impulsions fortes de rephasage sont bien adaptées pour générer un écho en spectroscopie, elles induisent du bruit au moment de la lecture qui submerge le photon porteur d'information. Une alternative substituant les impulsions optiques par des impulsions de champ électrique (effet Stark) a été proposée pour produire l'écho d'un photon sans bruit. Cette proposition a été démontrée expérimentalement avec des 
ions de praséodyme inclus dans une matrice cristalline d'orthosilicate d'yttrium [4]. D'une part, le faible niveau de bruit a été confirmé, mais de façon plus éclatante l'efficacité était de $69 \%$, ce qui est remarquable pour ce genre de réalisations.

L'adéquation entre la technique d'écho et les solides dopés permet d'exploiter par ailleurs les larges bandes passantes de ces matériaux. L'équipe du Laboratoire Aimé Cotton a par exemple montré qu'un cristal de YAG dopé au thulium (figure 1) pouvait enregistrer plus de 1000 impulsions en parallèle [5]. Cette démonstration avec une efficacité certes réduite (environ $1 \%$ ici) montre en tous cas la capacité multimode temporelle de ce type de systèmes.

\section{Les mémoires quantiques, un avenir encore à écrire}

Ce domaine de recherche a ainsi connu au cours des dix dernières années un foisonnement d'idées nouvelles, mais le chemin est encore long pour obtenir dans un même système une grande efficacité d'écriture et de lecture, un long temps de stockage, ou encore la capacité de multiplexer l'information stockée. II n'est pas possible de dire quels matériaux au sens large seront les plus efficaces. Leur choix

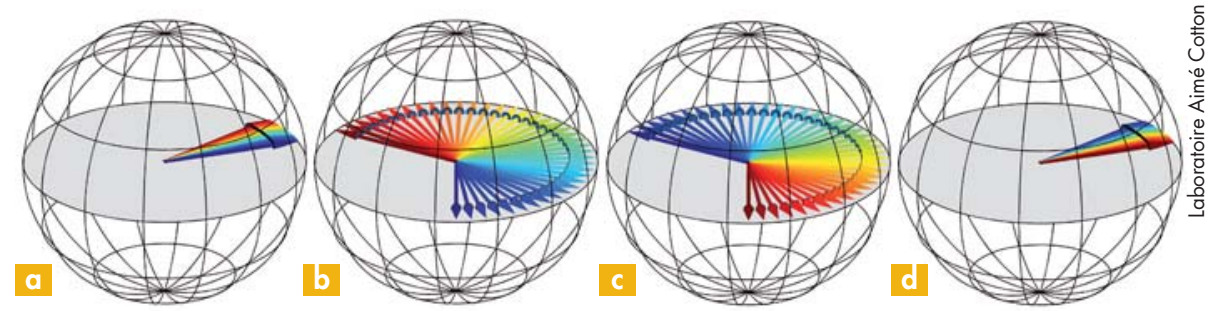

Figure 5. Représentation schématique d'une séquence d'écho de photon. Pour faire le lien avec la technique de résonance magnétique nucléaire, on représente les dipôles optiques comme des spins nucléaires en précession sur une sphère appelée sphère de Bloch. La couleur de chaque flèche indique une fréquence d'oscillation différente. Le déphasage accumulé au cours du temps à cause de l'élargissement inhomogène $s^{\prime}$ illustre par un étalement coloré sur le plan équatorial de la sphère. Après une excitation commune (a), les dipôles se déphasent fortement (b). L'impulsion de rephasage inverse la phase d'oscillation et permute de façon schématique les dipôles rouges et bleus (c). La séquence se termine par une remise en phase (d) et par la génération d'un écho.

dépendra très probablement de l'application. Cependant, ces premières réalisations permettent déjà de connecter de tels systèmes entre eux, ouvrant la voie à des fonctionnalités nouvelles en information quantique. Des premières expériences de réseau quantique sont apparues, comme nous l'avons vu avec l'expérience reposant sur des atomes uniques. Des segments de répéteur quantique, certes rudimentaires mais qui en présentent conceptuellement les fonctionnalités, ont également été démontrés avec des nuages d'atomes froids. De nombreuses années d'effort, expérimental et théorique, seront encore nécessaires mais les mémoires quantiques sont désormais une réalité !

\section{Références}

[1] http://quantumrepeaters.eu

[2] J. Laurat, Mémoires quantiques : Stocker l'insaisissable. Pour la Science n68, Dossier " Le Monde quantique », juillet 2010.

[3] A. Nicolas, L. Veissier, L. Giner, E. Giacobino, D. Maxein \& J. Laurat, A quantum memory for orbital angular momentum photonic qubits. Nature Photonics 8, 234-238 (2014).

[4] M.P. Hedges, J.J. Longdell, Y. Li \& M.J. Sellars, Efficient quantum memory for light. Nature, 465, 1052-1056 (2010).

[5] M. Bonarota, J.-L. Le Gouët \& T. Chanelière, Highly multimode storage in a crystal. New Journal of Physics, 13, 013013 (2011).

\section{- SPECTROGON}

\section{Filtres Interférentiels}

De 200 á 15000 nm

- Passe-bande

- Passe-haut

- Passe-bas

- Large bande

- Densité neutre

- Disponible en stock

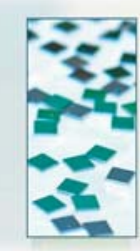

De 150 á 2000 nm

- Compression d'impulsion

- Télècom

- Accordabilité spectrale

- Monochromateurs

- Spectroscopie

- Disponible en stock 